\title{
Quand les mouvements sociaux changent le politique. Le cas du mouvement étudiant de 2012 au Québec.
}

\author{
Pascale Dufour \\ Université de Montréal \\ Louis-Philippe Savoie \\ Université de Montréal
}

\begin{abstract}
Résumé. À travers l'analyse du conflit étudiant du printemps 2012 au Québec, nous montrons comment les mouvements sociaux peuvent changer, temporairement, le politique. Le politique est entendu ici comme l'ensemble des règles et pratiques individuelles et collectives qui régissent les relations entre les acteurs à propos du gouvernement de la communauté. Plus précisément, nous montrons que le mouvement de contestation a transformé le politique de trois manières. Premièrement, les six mois du conflit étudiant ont créé un nouveau clivage autour duquel la vie politique s'est réorganisée. Deuxièmement, les acteurs partisans et les associations étudiantes ont modifié leurs pratiques et actions quotidiennes, redéfinissant leur mode de relations et leur politique d'alliance. Troisièmement, l'expérience prolongée de la mobilisation a changé le rapport au politique pour les individus mobilisés en bousculant les articulations usuelles entre l'usage de la politique institutionnelle et celui de la politique protestataire.
\end{abstract}

\begin{abstract}
Using the case of the 2012 student conflict in Québec, we show how social movements can temporarily transform politics. We define politics as the set of rules and individual and collective practices that regulate relations between actors regarding a community's government. We show three ways in which the 2012 student conflict transformed politics. Firstly, the six-month conflict created a new division around which politics reorganized itself. Secondly, political parties and student unions modified their daily practices, redefining their relationships and policies of alliance. Finally, the prolonged experience of mobilization transformed activists' relationship to politics by rearticulating the distinction between institutional and protest politics
\end{abstract}

«En un moment, la couleur rouge, comme autant d'étincelles jaillissant, de mains en mains et de poitrines en poitrines, courait sur des zones entières du quai, des rues, de la place de la Grève, et éblouissait ou consternait les regards des spectateurs placés aux fenêtres de 1'Hôtel de Ville ». (Alphonse de Lamartine, cité par Riot-Sarcey, $2012: 133$ )

De manière dominante, l'étude des mouvements sociaux, particulièrement l'approche dite du processus politique, a tenté de montrer comment les contextes institutionnels et politiques 
avaient une influence sur la forme, le contenu, la durée et l'efficacité des mobilisations. Dans cet article, nous proposons de renverser la perspective et de voir comment un mouvement social d'envergure, le mouvement étudiant québécois de 2012, a modifié temporairement le politique, entendu comme l'ensemble des règles et pratiques individuelles et collectives qui régissent les relations entre les acteurs collectifs et les individus à propos du gouvernement de la communauté. Le but de notre démarche n'est pas de soutenir que le mouvement social de 2012 a eu des conséquences politiques ultérieures sur le plan institutionnel, mais bien de considérer le moment de l'irruption sociale (ce que fait un mouvement social) comme un moment inhabituel de fonctionnement du politique et d'analyser ce moment-là précisément.

Après avoir examiné les principaux travaux sur les effets des mouvements sociaux (partie I), nous montrons que le politique a été transformé de trois manières. Premièrement, les six mois du conflit étudiant (entre février et l'élection québécoise du 4 septembre 2012) ont créé un nouveau clivage autour duquel la vie politique s'est réorganisée, l'ordre du jour des acteurs institutionnels (principalement les partis politiques) ayant été imposé par le bas (les mobilisations) (partie II). Deuxièmement, les principaux acteurs collectifs concernés par le conflit (acteurs partisans et associations étudiantes) ont modifié leurs pratiques et actions quotidiennes, redéfinissant leur mode de relations et leur politique d'alliance (partie III). Troisièmement, l'expérience prolongée de la mobilisation a changé le rapport au politique pour les individus mobilisés en bousculant les articulations usuelles entre l'usage de la politique institutionnelle et celui de la politique protestataire (partie IV). 


\section{Quand les mouvements sociaux font de la politique}

La sociologie des mouvements sociaux n'offre pas de réponses claires à la question des effets politiques des mouvements sociaux (Ancelovici et Dupuis-Déri, 2014 : 357). Plus généralement, l'approche du processus politique s'est longtemps concentrée sur les déterminants de l'action collective et ce n'est que récemment que des travaux ont abordé la question des effets ou des conséquences des mouvements de front. Par exemple, Didier Chabanet et Marco Giugni (2010 : 8) répertorient six grands types de conséquences possibles. Ce sont : les effets politiques internes au mouvement (changements dans les rapports de force du mouvement) ; les effets politiques externes, qui vont de l'acceptation d'un acteur social comme porte-parole légitime d'une catégorie de population, à l'acceptation par le gouvernement des revendications, à des changements institutionnels importants (lois ou règlements); les effets culturels internes (changement de valeurs) et externes (opinion publique et attitudes dans la société) ; et les effets biographiques internes (changement dans le mode de vie des participants) ou externes (changement dans le mode de vie de la société). Malgré de très fortes difficultés méthodologiques, la recherche à ce propos a connu une accélération depuis les années deux mille et a tenté de mesurer la nature des changements que les mouvements sociaux pouvaient provoquer et les conditions les plus favorables à leur réalisation.

Edwin Amenta, Neal Caren, Elizabeth Chiarello et Yang Su (2010) proposent une synthèse des travaux récents. Certains auteurs démontrent l'importance des mouvements sociaux pour le politique (Baumgartner et Mahoney, 2005 ; Piven, 2006) alors que d'autres minimisent leur rôle (Skocpol, 2003 ; Burstein et Sausner, 2005 ; Giugni 2007). Au-delà des débats qui traversent ce sous-champ, leur synthèse démontre que « [t]here is conclusive evidence from wellcrafted studies that the largest movements have had political impacts on some issues of concern 
to them. Scholars who ignore movements in analyses of political issues over which large movements are contending do so at their peril » (Amenta et coll., 2010 : 295).

Cependant, il demeure difficile d'évaluer l'influence des acteurs protestataires par rapport à d'autres acteurs investis sur les mêmes enjeux. De plus, les mécanismes concrets permettant cette influence demeurent très imprécis. Les recherches récentes ont exploré les conditions qui favorisent l'action des mouvements sociaux en mettant en relief la nécessité de considérer les interactions entre le contexte politique et les conditions de l'action.

Dans cet article, nous explorons une piste distincte, qui vient compléter et préciser la littérature existante, en partant de l'intuition suivante : certains contextes extraordinaires, comme une mobilisation sociale d'envergure, peuvent créer (ou contraindre) de nouvelles opportunités d'alliances et modifier, chemin faisant, le rapport des acteurs aux différentes actions. Dans une période de forte turbulence sociale, les règles du jeu politique sont temporairement modifiées et de nouvelles pratiques peuvent se mettre en place, créant de nouvelles dynamiques entre les acteurs et les actions. Dans cette perspective, les facteurs structurels (ressources, structures des opportunités politiques et habitus des organisations) qui contraignent généralement et normalement les actions collectives sont temporairement suspendus, ou, potentiellement, moins déterminants. C'est sur ce moment de turbulence que nous concentrons notre analyse, montrant comment un mouvement social peut changer le politique, au moins temporairement. Pour être encore plus clair, il ne s'agit pas de mesurer l'effet des mobilisations sur le système et les dynamiques politiques québécoises à plus long terme, mais bien de considérer le moment des mobilisations lui-même comme un moment de redéfinition du politique. Le cas québécois nous permet de réfléchir à une interrogation simple, mais peu abordée : au-delà de la question des effets ou des résultats politiques, au-delà de la refondation de l'action que les mouvements 
sociaux permettent (ce que la littérature nomme les effets culturels), que se passe-t-il du côté du politique quand les mouvements sociaux font la politique?

Les mobilisations du printemps 2012 au Québec constituent un terreau extrêmement fertile pour vérifier cette intuition. En effet, il est très rare dans les démocraties représentatives d'Europe de l'Ouest ou d'Amérique du Nord qu'un épisode de mobilisation de masse dure assez longtemps pour créer le type de moment évoqué. Les événements de mai 1968 en France et les mobilisations grecques contre l'austérité amorcées en 2011 constituent deux exceptions. Les mobilisations de 2012 au Québec ont été visibles au cœur de l'espace public de février à septembre de cette année-là. Durant cette période, les partis politiques et les acteurs sociaux ont construit des relations dans un contexte extraordinaire et suffisamment longtemps pour qu'on puisse les observer et qu'elles se catalysent en « routines de crise ». Dans la plupart des conflits sociaux, au contraire, la durée courte du conflit ne permet pas la répétition dans les pratiques des acteurs. Nous proposons une analyse qui se situe à la fois au niveau des acteurs collectifs (partis politiques et associations étudiantes) et des militants. Pour eux aussi, la durée de l'implication est exceptionnelle et a permis l'établissement de « routines de mobilisation » tout en maintenant une intensité d'engagement très élevée. Il est raisonnable de supposer que cette situation a provoqué des conséquences biographiques importantes et que nous serons à même de repérer des changements de rapport au politique chez les individus.

De nombreux articles, chapitres et ouvrages ont été publiés à propos du « printemps québécois ». Pour la plupart, il s'agit de témoignages de participants, d'analyse à chaud des événements ou de réflexions sur les dynamiques internes des luttes (entre autres Brisson, 2012 ; Isabel et Théroux-Marcotte, 2012 ; Argument, 2013 ; Nadeau-Dubois, 2013 ; Poirier St-Pierre et Éthier, 2013 ; pour des bibliographies plus exhaustives, voir Marcil, 2014 ; L'Oreille tendue, 
2014). Ils témoignent de la nature peu ordinaire des événements de 2012. Peu d'écrits ont, jusqu'à présent, proposé un examen plus approfondi des mobilisations. L'ouvrage récent dirigé par Marcos Ancelovici et Francis Dupuis-Déri (2014) reste le seul pour l'instant à poser les premiers jalons d'une analyse d'une des plus grandes crises politiques que le Québec ait connu au cours des soixante dernières années. Dans la très grande majorité des contributions de l'ouvrage, les auteurs mentionnent le caractère singulier de la crise, notamment en ce qui concerne la durée, l'intensité et le nombre de personnes mobilisées.

En plus de cette littérature secondaire, notre analyse s'appuie sur des entrevues semidirectives. Nous avons rencontré (janvier et février 2013) les porte-paroles des trois principales associations étudiantes impliquées dans le conflit et réalisé neuf entretiens avec des militants. Les militants qui se sont prêtés à ces entretiens présentent des profils variés. Cinq répondants ont 21 ans ou moins, trois ont entre 22 et 26 ans et un, à 36 ans, est plus âgé que la moyenne des étudiants. Six répondants sont des hommes, trois sont des femmes. Cinq répondants étaient inscrits dans un Collège d'enseignement général et professionnel (cégep) durant le conflit étudiant et quatre étaient inscrits à l'université (principalement à l'Université de Montréal). Six répondants sur neuf poursuivaient un cursus en sciences humaines ou sociales. Tous les répondants sauf un ont vécu la grève à Montréal. Finalement, tous nos répondants sauf deux ont voté à l'élection provinciale du 4 septembre 2012 : des deux qui n'ont pas voté, un l'a fait par choix et l'autre parce qu'il n'avait pas la citoyenneté canadienne. Ces entrevues n'ont aucune prétention à l'exhaustivité, ni à la représentativité de la diversité des situations possibles. L'absence d'étudiants de l'Université du Québec à Montréal, d'étudiants des universités anglophones et des régions limite fortement la diversité de notre échantillon. Elles permettent tout de même d'esquisser un premier portrait—qui restera à compléter par une recherche plus 
poussée - du processus de construction du rapport au politique qui s'opère en temps de crise. Nous avons également utilisé, pour l'analyse des institutions d'enseignement en grève, des données quantitatives fournies par la Fédération étudiante universitaire du Québec (FEUQ), qui a maintenu jusqu'au début du mois d'avril une base de données comprenant l'ensemble des associations étudiantes en grève. Nous avons ensuite contre-vérifié et complété ces données à l'aide de diverses ressources : les sites web des associations étudiantes, le site Www.greve2012.org ainsi que certains journaux régionaux.

Dans la partie qui suit, nous montrons comment les acteurs du mouvement de 2012 ont modifié le cours normal des choses et imposé un clivage politique qui a réorganisé la vie politique québécoise durant six mois.

\section{Un scénario exceptionnel : les acteurs du conflit du printemps 2012 modifient le scénario}

Le conflit étudiant de 2012 est unique par son envergure et il est nécessaire de revenir sur son déroulement. La première section présente brièvement les acteurs collectifs qui ont joué à l'avant-scène de ce conflit. Puis, nous retraçons les moments et événements clefs qui ont contribué à créer un nouveau clivage.

Les acteurs et leur mise en scène : la création d'un front uni des associations étudiantes

Le mouvement étudiant est un acteur important de la vie politique québécoise depuis au moins les années soixante (Colas, 2008). Il s'agit toutefois d'un acteur jusqu'à récemment peu étudié ${ }^{1}$. Les associations étudiantes sont depuis les années quatre-vingt encadrées par la Loi sur l'accréditation des associations d'élèves et d'étudiants (chapitre A-3.01). Ce cadre législatif, fortement inspiré du Code du travail qui encadre les activités syndicales au Québec, est unique 
au Canada (Brunelle, Lampron et Roussel, 2012 ; Rousseau, 2013 ; Makela et AudetteChapdelaine, 2014). Il assure une autonomie aux associations étudiantes et des sources de financement stables et récurrentes tout en imposant certaines contraintes démocratiques (pour le détail, voir Makela et Audette-Chapdelaine, 2014).

En 2012, quatre associations nationales sont présentes sur la scène du conflit. La Fédération étudiante universitaire du Québec (FEUQ), créée en 1989 dans le contexte du dégel des frais de scolarité imposée par le gouvernement libéral de Robert Bourassa, regroupe 15 associations et 125000 étudiants universitaires. Elle est fondée à une époque où la plupart des associations universitaires ne sont membres d'aucune association nationale. La Fédération étudiante collégiale du Québec (FECQ), créée en 1990 dans le même contexte, regroupe 23 associations étudiantes et 80000 étudiants de cégep. Ces deux associations ont l'habitude de travailler conjointement, et malgré des difficultés, ont maintenu une collaboration étroite tout au long du conflit de 2012, comme elles le font généralement (Colas, 2008). Ces organisations sont principalement animées d'une volonté d'amélioration des conditions de vie et d'études de leurs membres, et plus largement des étudiants québécois, comme le révèlent leur mission respective. Elles se positionnent aussi comme des groupes jeunes et interviennent sur une variété de sujets². La Coalition large de l'ASSÉ (CLASSE) est une coalition temporaire formée autour des structures de l'Association pour une solidarité syndicale étudiante (ASSÉ). Au terme du conflit, elle regroupe 103000 étudiants dans 67 associations étudiantes. L'ASSÉ a été formée en 2000 dans le contexte de la lutte contre la création de la Zone de libre-échange des Amériques (ZLÉA), de la lutte à la taxe à l'échec au collégial et suite à la dissolution du Mouvement pour le Droit à l'Éducation (MDE) (Lacoursière, 2007). Elle s'identifie au syndicalisme de combat, dans la lignée de l'Union générale des étudiants du Québec (UGEQ), de l'Association nationale des 
étudiantes et étudiants du Québec (ANEEQ) et du MDE (Theurillat-Cloutier, Leduc et Lacoursière, 2014 : 51). L’ASSÉ regroupe des étudiants universitaires et collégiaux et milite pour la gratuité scolaire. Elle adopte des modes de fonctionnement plus décentralisés, notamment inspirés des pratiques des réseaux d'affinités et libertaires nés dans la mouvance altermondialiste (valorisation de la démocratie directe et de l'horizontalité dans la prise de décision, nomination de porte-parole en lieu et place d'un président). Durant la grève, la CLASSE tentera de maintenir cette exigence de démocratie participative et directe (Ancelovici et Roy-Allard, 2014). L'ASSÉ favorise également la construction de liens de solidarité avec d'autres acteurs collectifs nationaux (les milieux syndicaux et le milieu communautaire) et prend position sur des enjeux sociaux qui dépassent les questions directement en lien avec la vie étudiante. La CLASSE adoptera la même position durant le conflit, en travaillant notamment avec la Coalition opposée à la tarification et la privatisation des services publics.

À cela s'ajoute également la Table de concertation étudiante du Québec (TaCEQ), fondée en 2009 par des associations étudiantes qui avaient quitté la FEUQ au sortir du conflit étudiant de 2005 (Theurillat-Cloutier, Leduc et Lacoursière, $2014: 51$ ). Elle regroupe quatre associations et 65000 étudiants mais a été peu présente dans le conflit de 2012. Notamment, ses membres ont été beaucoup moins enclins à faire la grève que les membres des trois autres organisations étudiantes. L'organisation cherche à se démarquer des autres associations nationales par une structure décentralisée qui souhaite laisser une place prépondérante aux associations étudiantes locales (TaCEQ, 2014).

Depuis les années deux mille, les relations entre les différentes associations étudiantes opposent la FECQ et la FEUQ à l'ASSÉ. Dès sa fondation, l'ASSÉ s'inscrit en rupture avec les pratiques des fédérations étudiantes qu'elle juge peu combatives et peu démocratiques (entrevue, 
porte-parole de la CLASSE). En 2005, lors de la précédente grève étudiante contre les compressions de 103 millions de dollars dans le régime des prêts et bourses, la division du mouvement étudiant était clairement apparue. En effet, à la suite d'une première rencontre, le gouvernement libéral de l'époque avait exclu la CASSÉE (la Coalition de l'ASSÉ élargie fondée pour le mouvement de grève de 2005) de la table des négociations, arguant son refus de condamner la violence, condition acceptée par les deux autres associations (La Presse canadienne, 2005). Le conflit s'est terminé en demies teintes : les 103 M\$ ont été retournés au régime de prêts et bourses, mais en 2006-2007 uniquement. La FEUQ, suite à une résolution de son congrès, a appuyé l'entente, alors que la FECQ est demeurée officiellement neutre (ses membres l'ont toutefois entérinée) et la CASSÉE a dénoncé ce qu'elle a qualifié « d'entente à rabais » (ASSÉ, 2005).

En 2012, en revanche, un front uni a été voulu et maintenu dans l'espace public, même si de fortes tensions existaient entre les associations (entrevues avec les trois porte-paroles). Soulignons que le rapport de force entre les associations étudiantes a changé. La Figure 1 illustre la proportion d'étudiants en grève par affiliation nationale en 2005, alors que la Figure 2 présente ces mêmes proportions en 2012. La CLASSE atteignait 100000 membres en 2012 et entre 55000 (Baril, 2005) et 70000 en 2005 (Lafrance, 2005). On remarque que la FEUQ fédérait moins de membres en grève et une part importante (12\% des étudiants) était doublement affiliée, généralement à la CLASSE et une autre association nationale. Le rôle central de la CLASSE dans le conflit de 2012 n'est donc pas uniquement liée à une variation dans les taux d'affiliation des autres organisations, mais bien à un poids politique (et médiatique) sans commune mesure avec le conflit de 2005 . 


\section{[Insérer figure 1]}

\section{[Insérer figure 2]}

Le conflit de 2012 s'est traduit par de nombreuses manifestations « historiques » dans la métropole (notamment les manifestations du 22 mars, 22 avril et 22 mai), rassemblant des centaines de milliers de personnes durant plusieurs mois. Le mouvement social créé a clairement (et temporairement) bouleversé le jeu politique. Revenons sur quelques moments et évènementsclefs qui ont jalonné la construction et l'imposition d'un nouveau clivage politique dans l'espace public.

\section{La construction d'un clivage temporaire}

La notion de clivage présente la particularité de relier les divisions sociales et les divisions idéologiques (Bartolini, 2005 : 4). Ainsi, un clivage n'est pas seulement un conflit d'idées au sein d'une population, mais bien une ligne de démarcation ancrée dans une structure sociale et liée à une stratification sociale particulière. Par conséquent, le clivage droite-gauche ne signifie pas seulement, sur le plan politique, un ordonnancement différent des idées et des intérêts, mais aussi le fait que cette division soit liée à des groupes sociaux particuliers que nous pouvons identifier.

Le conflit étudiant de 2012 a provoqué la construction d'un clivage politique temporaire autour duquel s'articulaient des idées et des intérêts distincts et qui divisait aussi la population en deux camps. Rappelons les phases du conflit qui marque la construction de ce clivage. ${ }^{3}$

La première phase, la phase de mobilisation, construit le rapport de force avec l'État. Elle commence plus d'un an avant les grèves de 2012, lors de l'annonce le 17 mars 2011 par le 
gouvernement du Québec des modalités de la hausse des frais de scolarité qui devait entrer en vigueur à l'automne 2012 (Ministère des Finances, 2011). Il s'agissait d'une hausse de 75 pour cent (soit $1625 \$$ ) sur cinq ans. Plusieurs mobilisations contre la politique gouvernementale sont alors organisées, mais l'histoire du printemps 2012 commence réellement avec la manifestation conjointe des quatre principales organisations étudiantes (FECQ, FEUQ, ASSÉ, TaCEQ) du 10 novembre 2011, regroupant près de 30000 étudiants. Suite à cette mobilisation, l'ASSÉ fonde une coalition: la Coalition large de l'Association pour une solidarité syndicale élargie (CLASSE). La rumeur d'une grève au printemps prend forme. Le 13 février 2012, les premières associations votent une grève générale illimitée (La Presse canadienne, 2012a) et elles seront suivies par des centaines d'autres. Le 22 mars, alors que 300000 étudiants sont en grève à travers le Québec (pour cette journée), une des manifestations les plus importantes de l'histoire de Montréal se déroule dans le centre-ville où 200000 personnes défilent (La Presse canadienne, 2012b). À partir de mars, le nombre de grévistes demeure relativement stable, oscillant entre 150000 et 200000 étudiants jusqu'en mai 2012. De son côté, le gouvernement n'a pas répondu à la grève avant le 29 mars (Savard et Cyr, 2014 : 62). Durant cette première phase, le blocage politique est total. Nous verrons dans la section suivante ce qu'il signifie pour le travail politique quotidien des acteurs concernés.

La deuxième phase, la période de judiciarisation du conflit, commence avec les injonctions qui opposent le droit (contractuel et individuel) de suivre des cours pour lesquels des frais ont été acquittés au droit politique de faire la grève. Le 30 mars, une première injonction est accordée par la Cour supérieure à une étudiante opposée à la grève. En tout, 63 injonctions seront prononcées, dont 57 demandées par des individus, les six autres étant demandées par une université (l'Université de Montréal ou l'Université du Québec à Chicoutimi) contre une ou des 
associations étudiantes (Makela, 2014 ; compilation des jugements d'injonctions recensés à l'aide de la base de données CanLII). Les injonctions ordonnées ont rarement été respectées, que ce soit par les établissements ou par les associations étudiantes, et se sont accompagnées de fortes tensions au sein des établissements, voire d'affrontements violents entre les services de police ou les services privés de sécurité et les étudiants.

Progressivement, le conflit politique est renvoyé aux tribunaux au lieu de demeurer dans l'arène politique. Outre les injonctions mentionnées, la répression policière s'accroît au mois d'avril et de mai ; certaines manifestations se soldent par des arrestations de masse (comme la manifestation du 4 mai à Victoriaville) (Radio-Canada, 2012) ce qui ajoute au phénomène de judiciarisation. Le 18 mai, le gouvernement du Québec adopte le projet de loi 78 (qui deviendra la loi 12), « Loi permettant aux étudiants de recevoir l'enseignement dispensé par les établissements de niveau postsecondaire qu'ils fréquentent » (2012, chapitre 12), une loi spéciale d'une année restreignant fortement le droit d'association et de manifestation.

L'adoption de cette loi va provoquer une « réaction populaire spontanée » (Penafiel, 2012) : tous les soirs, à $20 \mathrm{~h}$ entre le 18 mai (Bélair-Cirino, 2012) et la mi-juin ${ }^{4}$, des centaines de résidents sortent sur leur balcon, dans la rue, en tapant sur des casseroles, afin de défier la loi (Drapeau-Bisson, Dupuis-Déri et Ancelovici, 2014). Cette période constitue le moment où le conflit autour des enjeux étudiants devient très clairement un conflit social et où un clivage politique se forme. La mobilisation déborde la population étudiante et les enjeux de la hausse des frais ; dans les rues les manifestants et les marcheurs dénoncent les politiques du gouvernement en place et sa gestion de la crise. Plusieurs thèmes interreliés s'articulent autour de la question des frais de scolarité dont la légitimité collective du mouvement de grève et des associations étudiantes comme acteurs politiques, le recours au droit versus le recours à la négociation 
politique pour régler un conflit social, les droits politiques des citoyens versus leur droit de consommation (à travers les débats autour de la loi 12).

La troisième phase est la phase de démobilisation. Elle suit l'annonce au milieu du mois de mai par le gouvernement du report de la session au mois d'août 2012. Le gouvernement, qui se trouve face à une crise politique majeure, déclenche des élections qui auront lieu le 4 septembre. Défait, le gouvernement libéral de Jean Charest cède sa place au gouvernement péquiste minoritaire de Pauline Marois. Si les élections du 4 septembre ne marquent pas la fin de l'histoire, elles changent néanmoins la dynamique politique, suffisamment pour que nous en fassions un tournant du conflit auquel notre analyse s'arrête.

Il apparaît clairement à travers chacune de ces phases que le conflit étudiant constitue au fil des mois une véritable démarcation sociale, pour reprendre les termes de Stefano Bartolini (2005). Il est également sous-tendu par une division forte de l'opinion publique dont les positions sont liées à des caractéristiques sociologiques. Nous sommes bien en présence d'un clivage.

La grève étudiante et l'opinion publique : un sujet qui divise La population du Québec, quand elle est sondée sur ce conflit, apparaît extrêmement divisée. La mesure la plus constante tout au long du conflit est tenue par Léger Marketing pour le compte du Journal de Montréal. La Figure 3 présente les résultats pour la période du 23 février au 21 mai.

\section{[Insérer figure 3]}

On peut voir que les étudiants démarrent le conflit avec un capital de sympathie relativement important : la population est divisée à parts égales entre l'appui au gouvernement du 
Québec et l'appui aux étudiants. L'appui décroit jusqu'au sondage du 28 mars, qui suit directement la grande manifestation du 22 mars. La période suivante, marquée par les premières offres de négociations et plusieurs affrontements lors des manifestations, semble profiter au gouvernement : ses appuis atteignent 60 pour cent le 10 mai et ce malgré le fait que 71 pour cent des Québécois jugent que le gouvernement Charest a mal géré le conflit étudiant (Léger Marketing, 2012a).

Bien que peu de données permettent une analyse sociologique des soutiens et des opposants au mouvement étudiant, un sondage CROP (2012) mené entre le 22 et le 25 mai 2012 et comprenant un échantillon de 1500 personnes permet de brosser un portrait assez juste de l'état des lieux. Ce sondage intervient après l'adoption de la loi spéciale, au moment où l'appui aux étudiants est plutôt faible, à seulement 36 pour cent (contre $64 \%$ pour le gouvernement). On note une division géographique et politique : les opposants aux étudiants sont beaucoup plus nombreux dans la grande région de Québec, où seulement un répondant sur cinq appuie les étudiants. Il s'agit aussi de la région électorale la plus à droite au Québec (Bélanger et Nadeau, 2009 ; Drouilly, 2012 ; Nadeau et Bélanger, 2013). Les variations selon l'âge offrent des résultats peu surprenants : les plus jeunes, qui présentent aussi des revenus plus faibles, appuient davantage les étudiants (à $44 \%$ chez les 18-34 ans) alors que les personnes plus âgées appuient davantage le gouvernement ( $72 \%$ d'appuis chez les 55 ans et plus). Finalement, les francophones (38\%) appuient nettement plus les étudiants que les anglophones (23\%). À la lumière de ces données, il ressort clairement que les personnes plus âgées, les anglophones, et les citoyens provenant de la région de Québec sont les principales sources des appuis au gouvernement. Il s'agit dans tous les cas d'électorats plus conservateurs (Bélanger et Nadeau, 2009). 
Les dynamiques du conflit ont permis en fin de compte l'émergence d'un véritable clivage qui associait des positions politiques distinctes sur des enjeux non courants (droit politique, accessibilité des études) à des publics particuliers. Les acteurs collectifs au cœur du conflit, les partis politiques et les associations étudiantes ont dû réorganiser leurs pratiques quotidiennes autour de ce clivage temporaire. C'est à l'examen de ces ajustements qu'est consacrée la partie suivante

\section{Comment les associations étudiantes ont changé le politique}

Les entrevues effectuées révèlent que deux types de conceptions du rapport à la politique instituée coexistent en temps normal au sein du mouvement étudiant. Elles se situent dans un continuum entre une pratique de la pression-négociation, dont la FECQ et la FEUQ sont plus proches, et une seconde orientée vers la contestation sociale, dont l'ASSÉ se réclame.

La première conception est plus conforme aux règles du jeu institutionnel et consiste à adopter les pratiques classiques des groupes d'intérêt (Grossman et Saurugger, 2006). Ainsi, en temps normal, les associations entretiennent des relations régulières comprenant des contacts hebdomadaires avec les partis politiques et le gouvernement. Les organisations participent pleinement aux instances de consultation. Elles privilégient la négociation et leur action s'apparente au lobbying; les relations interpersonnelles avec les responsables politiques et les membres de la fonction publique concernés importent beaucoup dans le cadre de cette approche. «On entretenait des liens assez réguliers avec les partis politiques », nous confiera le président de la FECQ, «parce qu'on évaluait que les gens qui étaient le plus à même, souvent, de faire cheminer nos revendications, de transformer nos revendications en réalisations, c'était les pouvoirs publics. Donc on entretenait des liens tant avec les partis d'opposition qu'avec les 
cabinets ministériels ». Ce recours au lobbyisme n'exclut pas l'utilisation de pratiques protestataires, dont les manifestations et la grève, mais en module l'utilisation en les inscrivant dans une démarche de pression politique pour atteindre des objectifs prédéfinis.

La deuxième conception se situe aux antipodes de la première et correspond davantage à ce qui définit habituellement une organisation de mouvement social. Clairement située dans l'arène de la contestation sociale, l'organisation n'entretient pas de relations avec les partis politiques et en fait d'ailleurs une position de principe forte (entrevues porte-paroles QS et CLASSE). Elle intervient peu, ou de manière très ciblée, dans l'arène électorale et favorise des modes d'action plus conflictuels. « Pour l'ASSÉ », rapporte le porte-parole de la CLASSE, « il n'y a pas d'alliés dans la sphère parlementaire [...] C'est une position historique que l'ASSÉ a décidé de ne pas ouvrir, par souci de cohésion organisationnelle ». Quasi caricaturale, cette opposition devient particulièrement intéressante à analyser en période de conflit social majeur. En effet, au printemps 2012, les trois associations étudiantes se sont retrouvées dans l'arène de la contestation, dans une négociation avec le gouvernement du Québec et, finalement, dans une campagne électorale. Que devient alors le rapport au politique?

\section{Quand les associations étudiantes mènent le bal politique}

Pour la FEUQ et la FECQ, les changements principaux sont, bien sûr, liés à la situation de grève. Si avant le déclenchement de la grève, les relations avec le gouvernement libéral ne sont pas au beau fixe, elles sont, à tout le moins fonctionnelles, et les canaux de communication sont ouverts. Avec les débuts du conflit, ces canaux vont être complètement fermés durant plusieurs semaines (jusqu'à la fin mars) avant que le chef de cabinet de la ministre de l'Éducation, puis la ministre de l'Éducation elle-même se manifestent. Durant presque deux mois, le mouvement étudiant fait 
la une des journaux sans entretenir de liens avec les autorités gouvernementales (Influence Communication, 2013 ; entrevues avec les responsables nationaux des trois associations étudiantes) : c’est la période de blocage précédemment décrite.

Tout au long du conflit, des contacts très réguliers seront entretenus avec le Parti québécois, qui forme l'opposition officielle. Le Parti fait en sorte que ses députés posent des questions en chambre, fassent parler du conflit au sein de l'enceinte parlementaire et, dans la mesure du possible, fassent pression sur le gouvernement en place :

On leur parlait pour voir un peu quel effet la pression avec du côté du Parti libéral, parce qu'eux étaient un peu plus près pour voir quand il y avait des tensions. Notamment dans un caucus, il y a certaines choses qui peuvent filtrer que nous on ne peut pas vraiment savoir [...]. C'est beaucoup plus à l'hiver que ç'a commencé à être un peu plus crucial le travail qu'on faisait avec eux. Il y avait des questions en chambre pour lesquelles on travaillait directement avec l'opposition officielle. C'était Marie Malavoy qui était critique en matière d'éducation. On travaillait avec son bureau à elle pour les questions en chambre, on leur donnait une idée de voici, il va y avoir tel événement donc préparez vous, ça risque de rebondir et vice-versa. (entrevue, présidente de la FEUQ)

Pour la présidente de la FEUQ, le mouvement étudiant a conservé l'initiative par rapport au gouvernement jusqu'au mois d'avril en appliquant une stratégie de pression qui consistait à prendre ses distances du gouvernement, organiser de grandes manifestations et faire adopter et maintenir de nombreux mandats de grève

En avril, l'absence de réponse du gouvernement devient clairement plus problématique, la durée du conflit limitant de plus en plus la possibilité de maintenir l'intensité de celui-ci. 
Quand la ministre de l'Éducation, Line Beauchamp, contacte directement la présidente de la FEUQ à la mi-avril, l'exécutif de la FEUQ est plutôt soulagé, même si personne ne se fait d'illusion sur la volonté du gouvernement de négocier. Le président de la FECQ mentionne d'ailleurs qu'il était perturbant de voir que le gouvernement demeurait sur sa position initiale pour ne pas avoir l'air de céder quoi que ce soit au mouvement étudiant. Ce faisant, « il se peinturait un peu dans le coin, parce qu'il ne pouvait pas négocier » (entrevue, président de la FECQ).

Les contacts vont se poursuivre jusqu'au début de juin (avec de vives tensions), de tentative de négociations en tentative de négociations. Pour le président de la FECQ, la diversification des formes d'action, dont les manifestations nocturnes qui ont débuté avec les négociations et les manifestations de casseroles en opposition à la loi spéciale, marque un tournant important dans le conflit. En effet, ce ne sont plus les associations étudiantes qui ont le contrôle de la situation. Selon lui, une négociation dans un conflit étudiant de cette envergure se règle par un échange : une entente favorable sur le front des droits de scolarité contre la cessation des moyens de pression. Or, il y a véritablement une « perte de contrôle, je pense qu'on peut l'appeler comme ça, sur les moyens de pression directs, qui faisait en sorte que nous on savait que notre pouvoir réel était limité », avouera le président de la FECQ.

À compter du $1^{\text {er }}$ juin, le gouvernement et les ministères coupent complètement les contacts avec les associations étudiantes ; la solution électorale apparait de plus en plus évidente. Si l'arrivée des élections avait été envisagée comme une possibilité du côté de la FECQ et de la FEUQ, qui s'étaient préparées en conséquence avant même le déclenchement de la grève, la CLASSE s'est retrouvée dans une tout autre situation. À la CLASSE, les relations aux partis politiques et au gouvernement en place sont très distinctes. La culture politique d'indépendance 
face aux partis de cette coalition est fondée à la fois sur la défiance d'une partie des militants à l'endroit des partis et leur pouvoir d'instrumentalisation, mais aussi sur la défiance des militants envers la démocratie représentative et la valorisation inverse de la démocratie directe (entrevue, porte-parole de la CLASSE, avril 2013). Cependant, la CLASSE et l'ASSÉ ne sont pas simplement des organisations antiparti et antisystème dans le sillage des organisations nées avec les luttes antimondialisation (della Porta, 2013). De manière plus contingente, la coalition est aussi une opposition à la FEUQ et à la FECQ, les militants à l'origine de l'ASSÉ ne voulant pas que le militantisme étudiant ne soit qu'un tremplin vers les partis politiques, dont le Parti québécois (entrevue, porte-parole de la CLASSE). Ils reprenaient, de ce point de vue, une longue tradition du mouvement étudiant portée par l'Association nationale des étudiants du Québec au milieu des années soixante-dix et interrompue avec la création des fédérations.

Rompue aux règles du jeu de l'arène protestataire, la CLASSE a joué un rôle central sur le terrain des mobilisations de rue et des multiples actions directes qui ont jalonné le conflit. Mais malgré sa participation au front uni, la CLASSE n'a pas bougé de sa ligne initiale par rapport au monde partisan, et n'a donc pas entretenu de relations avec les partis politiques, ni participé à des rencontres, à l'exception des négociations avec le gouvernement du Québec. En même temps, les trajectoires des militants se sont énormément croisées sur le terrain, notamment pour Québec solidaire (QS) et la CLASSE (y compris pour les porte-parole). Il n'y avait donc pas de rapport formel, certainement pas de rapport organique, mais les circonstances ont fait que les rapports informels ont été quotidiens, particulièrement entre militants d'allégeances diverses qui se rencontrent lors d'actions ou de manifestations. Cette situation, peu problématique en dehors de situations de conflit, devient plus difficile à gérer en situation de grève parce qu'elle rend beaucoup plus floue la question des alliés. Ainsi, l'ASSÉ a été une des six organisations 
fondatrices de la Coalition opposée à la tarification et la privatisation des services publics (surnommée « Coalition main rouge »). Dans ce cadre, elle a développé beaucoup de liens avec d'autres organisations de mouvements sociaux, qu'elle considère de longue date comme des alliés. Cependant, en situation de crise sociale comme au printemps 2012, les alliés sont aussi les militants des manifestations et des mobilisations qui peuvent faire partie de QS, un parti politique.

Pour le porte-parole de la CLASSE, la position d'indépendance de cette organisation face aux partis politiques, inscrite en quelque sorte dans le « code génétique » de l'ASSÉ, l'a empêchée d'investir l'arène électorale. À partir de l'annonce des élections, la CLASSE, en perdant l'initiative, aurait perdu du terrain ; elle n'a pas été capable de prendre une position (voter ou s'abstenir) qui lui aurait permis de poursuivre la lutte sur un autre terrain (entrevue, porte-parole de la CLASSE).

La situation prolongée de confrontation entre les acteurs collectifs du mouvement étudiant et le gouvernement a modifié les pratiques internes des associations étudiantes. Pour la FEUQ et la FECQ, plus habituées aux situations de négociation, la rupture de la communication avec les instances gouvernementales a créé de fortes incertitudes sur le choix des stratégies à adopter et posé la question du contrôle de la « politique de la rue ». Pour la CLASSE, la participation au front uni signifiait une présence aux tables de négociation et une réflexion parfois douloureuse sur la question des alliances, en particulier avec le parti politique QS, ainsi que des stratégies à adopter au moment du déclenchement des élections. Cependant, une forme de division du travail politique s'est opérée entre les associations étudiantes : la FECQ et la FEUQ demeurant en contact régulier et travaillant avec l'opposition officielle à l'assemblée nationale, la CLASSE gardant davantage l'initiative sur le terrain des mobilisations. 
Les acteurs politiques partisans ont également modifié leur manière de faire la politique durant ces six mois hors-norme, et ils ont dû suivre le rythme et les règles (informelles) d'un conflit dont ils n'avaient pas les rennes.

\section{Des remous sur la scène politique}

Très rapidement, les partis politiques ont été interpellés par le conflit étudiant de 2012. Le Parti québécois (PQ), faisant entre autres suite aux pressions étudiantes, s'était engagé dans son programme politique à revenir au gel des frais de scolarité au niveau de 2012, le temps de tenir un sommet sur l'enseignement supérieur (Parti québécois, 2011). Dès le déclenchement du conflit, les députés du Parti québécois appuieront les étudiants, d'abord sur la hausse de droits de scolarité et ensuite contre la loi spéciale. Durant le conflit, et ensuite en campagne électorale, le PQ proposera une indexation des droits de scolarité (Drouin, 2012) suivant l'évolution du revenu disponible des ménages, soit $3 \%$ par année (environ 70 \$ par année ; Gouvernement du Québec, 2013). Le PQ formant l'opposition officielle, il sera l'allié principal sur le plan de la politique partisane pour les responsables de la FECQ et de la FEUQ.

Ainsi, dès le début du conflit, le PQ intervient sur cet enjeu en chambre, apportant « la rue » dans l'arène institutionnelle ${ }^{5}$. Plus le conflit s'accroît en intensité, plus l'enjeu s'impose au politique. La Figure 4 présente l'évolution du travail parlementaire du PQ tout au long du conflit étudiant. Les deux catégories d'analyse retenues sont les blocs de questions posées lors de la période de questions et réponses orales, qui est la période la plus médiatisée des travaux parlementaires (Montigny et Pelletier, 2009), et tous les autres travaux parlementaires (principalement les débats sur des motions de censure et sur les projets de loi).

[Insérer figure 4] 
On constate que la grève étudiante a transformé l'ordre du jour des travaux parlementaires à l'Assemblée nationale. À eux seuls, les députés du PQ ont posé 73 questions principales au gouvernement ${ }^{6}$. Les événements déterminent de manière importante le volume de questions posées. À partir du mois d'avril, quand la possibilité de négociations directes entre le gouvernement et les associations étudiantes s'ouvre, on note une forte accélération. Le spectre de l'adoption d'une loi spéciale a aussi marqué les travaux parlementaires, avec 23 questions posées dans les deux semaines entourant la loi spéciale. D'autres outils parlementaires ont aussi été utilisés pour interpeller le gouvernement, dont trois motions de censure comprenant 16 interventions de députés du PQ. L'adoption de la loi 78 a été un moment particulier, comprenant un total de 25 interventions de tout type concernant le conflit étudiant. Les intervenants déployés révèlent aussi l'importance croissante du conflit : la chef du PQ commence à intervenir en chambre sur la question de manière hebdomadaire (à l'exception d'une semaine) dès la fin de mars. Il s'agissait d'ailleurs d'un objectif visé par les fédérations étudiantes. Selon la présidente de la FEUQ, « ça avait beaucoup plus de poids que la critique de l'opposition officielle ». Finalement, à partir de la mi-avril, la période de questions à l'Assemblée nationale s'ouvre presque toujours sur le thème du conflit étudiant et ce, pendant plus d'un mois.

Le parti de gauche Québec solidaire, créé en 2006, a été le premier allié du mouvement étudiant. En effet, défendant dans son programme des positions proches de celle de la CLASSE, il a dès le départ pris le parti des étudiants en grève. Si le travail parlementaire de QS a été restreint durant la période étudiée (n'ayant qu'un seul député), sa vie interne a été bouleversée. L'agenda de la grève étudiante et des mobilisations étudiantes s'est imposé à la formation politique. En plus des manifestations auxquelles les militants de QS ont activement participé, le 
parti a tenu des réunions extraordinaires de son comité de coordination à deux reprises (sur la question de la dénonciation de la violence politique et de la désobéissance civile). Un porteparole de QS constate en entrevue :

Je dirais que le printemps érable nous a forcés comme parti à... une réflexion pas facile, intense, émotive, où viennent se contrecarrer, plusieurs idées. Par exemple on va dire «mais oui, il y a des circonstances dans la vie d'un peuple où [la désobéissance civile] est nécessaire et l'histoire en est pleine ». D'autres qui disent « oui, mais c'est pas à un parti politique de proposer ça. Parce que comme parti politique, on aspire à faire des lois ; est-ce qu'on est train de dire aux gens, alors que nous voulons un jour être un gouvernement qui fera passer des lois, si les lois qu'on passe ne vous satisfont pas, vous avez juste à les défier ?» C'était le genre de discussions qu'on avait. Pour finalement en arriver à une position qui se résume ainsi : « nous n'appellerons pas nous mêmes à la désobéissance civile pacifique mais nous ne la condamnerons pas ».

Outre le programme, ce sont les normes de relations qui sont modifiées. Pour QS, il est intéressant de voir que les liens qui se sont créés avec le mouvement étudiant l'ont été « par la base ». Ils se sont structurés dans les manifestations, dans les établissements en grève, par l'intervention de porte-paroles ou des militants de QS qui vont parler aux étudiants dans les établissements universitaires (entrevue, porte-paroles de QS). Les règles du jeu politique sont chambardées : ce qui devient quotidien durant la grève étudiante, c'est le piquet de grève, la manifestation ou encore l'occupation des cégeps. Toutes les autres scènes politiques « normales » passent alors au second plan. 
Les associations étudiantes (et leurs mobilisations) ont créé un moment politique exceptionnel où le jeu est mené par des acteurs sociaux contestataires durant six mois, alors que les partis politiques jouent un rôle plus effacé, du moins jusqu'au déclenchement des élections. Le soir du 4 septembre, le PQ accède au pouvoir et forme le nouveau gouvernement (minoritaire). Il reprend alors le contrôle sur le processus politique : rapidement, il annule la hausse libérale des frais de scolarité et convoque un Sommet sur l'éducation, ainsi que son programme électoral l'avait annoncé. Dans cette nouvelle phase du conflit, les dynamiques entre les acteurs reprennent leur caractère coutumier : la FEUQ et la FECQ au sein du jeu institutionnel, l'ASSÉ en dehors. Le moment du Sommet sur l'éducation en offrira d'ailleurs une belle illustration ; l'ASSÉ refusera de participer aux discussions, après toutefois avoir participé à ses rencontres préparatoires, et le mouvement étudiant dans son ensemble apparaîtra à nouveau divisé, un peu comme en 2005.

Des bouleversements sont également apparus dans les trajectoires biographiques des militants, un domaine particulièrement peu étudié dans la littérature (Chabanet et Giugni, 2010 : 28). En effet, se mobiliser de manière intensive ou soutenue pendant six mois change certainement la vie quotidienne. Cela change également le rapport au politique.

\section{Comment le mouvement étudiant a changé le rapport au politique de ses militants}

Sur le plan empirique, le lien entre « voter avec ses pieds » (Fillieule et Tartotowsky, 2008) et déposer un bulletin dans une urne est confirmé. Selon les données de l'Enquête sociale générale de Statistique Canada (2008), les répondants qui ont manifesté rapportaient avoir voté à 84,4 pour cent, contre 78,7 pour cent pour les non-manifestants. Aux États-Unis, Alan Schussman et Sarah Soule (2005) suggèrent que les manifestants sont plus intéressés par la politique, plus informés, ont une meilleure perception de leur capacité de changer les choses 
politiquement, sont davantage à gauche du spectre politique et s'inscrivent plus souvent pour voter. La relation se vérifie aussi à l'échelle européenne. En 2012, à partir des données de l'étude EUROPART, composée de focus groups et de questionnaires auprès de 8030 répondants âgés de 15 à 25 ans, Rengard Spannring (2012:41-42) trace les contours de l'engagement politique des jeunes Européens. Elle montre que toutes les formes d'engagement sont corrélées entre elles : un jeune qui s'implique dans une ONG a plus de chance de voter, par exemple. On pourrait donc s'attendre à ce que la grève de 2012 ait eu un effet de politisation sur les jeunes mobilisés. Nos entrevues montrent que des mouvements complexes se sont tissés entre les différentes formes d'action politique et qu'à court terme (les entrevues ont eu lieu à l'hiver 2013), le rapport aux institutions représentatives pouvait, parfois, être compromis.

Pour certains des étudiants rencontrés, particulièrement les plus jeunes, l'expérience de la grève constitue leur premier engagement dans un conflit. Chez ceux-ci, le désir de s'engager était souvent présent, mais ce n'est qu'au cégep que l'opportunité de s'engager s'est présentée. Pour d'autres, déjà engagés, le moment de la grève constitue une période intensive et accélérée de l'apprentissage de lutte politique. Pour la plupart, c'est l'ensemble de leur vie qui s'organise autour du conflit. En cela, la période de la grève a créé un moment exceptionnel parce que durant une longue période, les contraintes quotidiennes changent radicalement : ce ne sont plus les études (ou la combinaison études-travail) qui rythment la vie, mais les besoins de la mobilisation. Pour plusieurs, l'implication a été quotidienne, ponctuée principalement par la participation à une ou plusieurs manifestations et une participation régulière aux assemblées générales locales, une activité qui vient avec des heures de discussion et de débats. Pour les personnes plus impliquées dans leur association locale, un travail de mobilisation est également au programme 
de chaque journée de grève. Plusieurs trajectoires ressortent de l'analyse de ces entrevues. Au moins deux grands portraits type se démarquent

\section{Le contestaire}

La figure du contestataire rassemble des étudiants déjà mobilisés ou des primo-militants qui ont vécu très intensément leur implication. «Ma maison, c'était mon école » (entrevue 9), nous raconte une militante. Entièrement absorbés par la vie d'étudiant en grève, ils participent à la mobilisation, aux actions dans et hors de leur institution scolaire chaque jour et pendant plusieurs mois. Le retour à la vie « normale », hors conflit, a été particulièrement difficile, malgré la poursuite de l'implication au sein de l'école (café étudiant) ou de l'association étudiante. Outre le changement de rythme imposé, ces contestataires ont de la difficulté à accepter le retour aux contraintes scolaires : comment « faire comme si rien ne s'était passé » alors que « tout », pour eux, s'est passé durant ce conflit. De même, le contestataire n'accepte pas l'issue du conflit : «Ce n'est pas vrai que les élections vont régler quelque chose ». Pour eux, au-delà de la question des frais de scolarité, c'est l'organisation de la société dans laquelle ils vivent qui doit être changé.

Le rapport aux institutions est ressorti transformé par l'expérience de la grève. L'expérience directe de la répression (arrestation lors d'une manifestation, matraquage, ou autres lors d'événements comme les manifestations de soir, la manifestation au moment du rassemblement pour le Grand Prix de Montréal, le Plan Nord, Victoriaville, le 15 mars, et ainsi de suite) constitue une rupture dans la trajectoire militante. Dans certains cas, l'implication devient plus forte, plus intense et se radicalise : « Mais en même temps vers la fin de la grève j'ai compris que ça ne me dérangeait plus de me faire arrêter, si c'est un geste politique [...]; ça ne donne rien si on s'empêche de faire des actions parce qu'on a peur de se faire arrêter » (entrevue 
9). Dans d'autres cas, l'implication se fait autrement pour éviter ce type d'affrontement, par exemple dans les assemblées générales, dans la mobilisation, voire dans les réseaux sociaux, plutôt que dans la participation à des manifestations : « J'ai fait deux manifs de soir et j'ai été victime de brutalité policière. Et ça, ça m’a traumatisé et depuis ce temps là, je n'ai plus été aux manifs de soir parce que ça me faisait trop peur » (entrevue 6). Ces expériences tendent aussi à développer une méfiance, voir une défiance active vis-à-vis de l'institution policière : « Le 7 mars, c'est la première manif où il y a eu vraiment de la brutalité policière, c'est comme si la pression avait vraiment monté. Après il y a eu le 15 mars avec des encerclements de masse. Moi c'était vraiment ma première expérience de brutalité policière. C'est à partir de ce moment là qu'il y a eu un déclic, je me suis rendue compte qu'on était dans un État policier » (entrevue 9).

Ces militants vont généralement garder une plus grande distance face aux partis politiques, bien que certains, même s'ils se définissent en opposition aux institutions, ont tout de même choisi de voter le 4 septembre, généralement pour QS : « Au moment de décider si je vote ou pas, je me suis dit, oui, je vais aller voter, en même temps je veux encourager la madame qui se présente pour Québec solidaire dans mon comté—moi, c'est un château-fort libéral » (entrevue 6). Autrement dit, même pour des personnes opposées au système économique et politique en place, l'abstention n'est pas un passage obligé (comme l'illustrait Luck, 2008). Dans un cas, jusqu'au dernier moment (le jour même des élections), la personne a hésité (entre le fait de voter et de ne pas voter) : «J'étais pas sûr jusqu'à la dernière minute en fait si j’irais voter ou pas. Je me disais s'il n’y a pas de campagne abstentionniste et si personne s'abstient, ça changera rien. Je suis aussi bien de donner mon vote à QS. Au final, je suis pas allé voter [...], je pourrais pas vraiment t'expliquer [...], j'ai vu la file et je me suis dit je veux pas faire ça, je voterai pas et 
tant pis. Au pire si le Québec sombre dans le chaos le Québec sombrera dans le chaos, je veux dire pour moi c'est pas tant une menace (entrevue 8).

Il serait intéressant d'approfondir l'analyse de ce positionnement militant hors des institutions (et parfois hors de la société) et la participation à des moments électoraux. Notamment, dans le cas du Québec, il se pourrait que la présence de QS, un parti dont l'histoire le situe à la jonction des urnes et de la rue, ait remplie cette fonction tribunicienne attribuée un temps au Parti communiste français (PCF) (Lavau, 1981). Comme le PCF dans les années soixante-dix, QS peut avoir jouer ce rôle d'intégration à la vie institutionnelle en permettant la médiation des intérêts et des identités des personnes qui se sentent mal représentées et peu entendues au sein des autres partis.

Le « paladin»

Le « paladin », selon le terme emprunté à un de nos répondants, est un militant déjà engagé avant le conflit de 2012 ou un primo-militant, qui va s'impliquer de manière soutenue durant le conflit et qui va prolonger cet engagement dans l'arène institutionnelle, à partir du déclenchement de la campagne électorale. Le moment de la grève a été l'occasion d'une prise de conscience des liens existants entre des enjeux (les frais de scolarité), des solutions politiques (la revendication de gratuité scolaire) et des processus sociopolitiques plus larges (la question de la marchandisation de l'éducation). Pour ce profil de militant, la poursuite du combat sur le terrain électoral devient nécessaire :

Je pense que j'ai arrêté de marcher fin juillet. Je suis allé à celle du 22 août mais fin juillet, j'ai arrêté. Quand on a su que finalement, les élections allaient être déclenchées, il allait y avoir une campagne électorale. Moi ça m'a arrêté, dans le sens que l'objectif était de les débarquer, et ça a été fait. Line Beauchamp a été la première, après ça a été le 
gouvernement au complet. Là moi, je jugeais que c'était fini. J'ai passé pour un vendu, dans le sens que « tu t'en vas en politique, c'est justement ce qu'on critique ». Un moment donné c'est quoi que vous voulez faire ? Je voyais pas comment cette implication-là, ce réveil, c'est un éveil aussi, c'était quoi la suite logique ? Moi je trouvais que c'était ça [l'implication électorale]. (entrevue 5)

Cet engagement subséquent au conflit, très intense (autant que durant la grève ou presque selon les propos tenus), peut être vécu sur le mode conjoncturel (il faut tout faire pour ne pas que les libéraux soient réélus) et s'arrêter tout de suite après l'élection du 4 septembre ou au contraire, se poursuivre au sein des partis politiques (jusqu'à être candidat aux élections). Avec les paladins, le tempo d'engagement est différent : la campagne électorale est clairement perçue comme un prolongement normal du conflit qui opposait les étudiants au gouvernement en place ; le soir du scrutin marque dans leur esprit la fin d'une étape et le début d'une autre. On retrouve, ici, la temporalité construite et perçue par la FEUQ, pour qui le 4 septembre 2012 marque le démarrage d'une nouvelle ère et le retour aux pratiques usuelles du militantisme étudiant.

L'après-conflit peut se traduire pour les personnes par la poursuite de l'engagement dans la sphère politique traditionnelle, mais il peut également se solder par un désengagement au bout de quelques mois. Certains paladins rencontrés se disent très déçus du parti au pouvoir qu'ils soutenaient activement. Pour certains, le désengagement est complet alors que pour d'autres, il concerne seulement l'arène électorale et se poursuit dans l'arène des conflits sociaux. Il faut, évidemment, prendre ces observations avec précaution : rappelons que les entrevues ont été menées juste après la tenue du Sommet sur l'éducation, qui a été vécu comme une grande déception par de nombreux étudiants engagés au printemps 2012. Pour mesurer la suite de la trajectoire avec précision et les articulations complexes de l'engagement/désengagement avec le 
parcours de vie, il serait nécessaire de faire des entrevues à plus long terme. On peut néanmoins conclure de cette indétermination de la poursuite de l'engagement, l'extrême fragilité du rapport aux organisations partisanes.

Pour une grande partie des personnes que nous avons rencontrées, l'expérience du militantisme durant la grève de 2012 a profondément marqué leur rapport au politique. L'élection a pris des sens divers, mais a toujours été vécue comme un moment important : soit comme le moment où la grève se conclut et la décision va se prendre, le lieu où le pouvoir politique s'exerce au final et où il faut s'impliquer, soit un moment où, au contraire, la grève échoue. D'autre part, nous avons noté des réactions diversifiées à des événements marquants : chez la plupart de nos participants, les événements de brutalité policière marquent un tournant dans leur expérience militante, mais les conséquences sur les trajectoires et les comportements sont variées (retrait, radicalisation, engagement différent). Le mouvement étudiant du printemps 2012 aura à n'en pas douter constitué un moment politique complexe pour les individus. Bougie d'allumage d'un conflit social d'envergure, il aura très certainement marqué une génération de militants. À cet égard, on peut soutenir l'hypothèse développée par Ancelovici et Dupuis-Déri (2014) : c'est dans les trajectoires biographiques que les effets de 2012 seront le plus durable.

\section{Conclusion : les frontières du politique redessinées}

Pour Diane Lamoureux, « [E]n 2012, il s’agit là d'un événement politique, au sens arendtien du terme. Il est en effet advenu quelque chose (il est encore trop tôt pour en déterminer tous les tenants er aboutissants) qui a interrompu le cours usuel des choses et qui a enclenché une dynamique dont l'issue était imprévisible » (2013:25). C'est précisément ce que cet article a tenté de faire : analyser comment le mouvement social qui a existé dans l'espace public entre 
février et septembre 2012 a redessiné, pour un temps, les frontières du politique. Partant d'une des caractéristiques fondamentales des mouvements protestataires, leur fonction d'irruption dans la vie politique normale, nous avons voulu regarder de plus près le fonctionnement quotidien de ce moment hors de l'ordinaire. Qu'en est-il ressorti ?

Pour les organisations (partisanes et les groupes étudiants), l'événement politique de 2012 (pour reprendre les termes de Lamoureux) a constitué un test d'élasticité : jusqu'où peut-on aller comme parti politique? Jusqu'où peut-on aller comme coalition ? En situation de crise sociale majeure, qui sont nos amis et nos ennemis? Quelles sont les actions politiques les plus appropriées ? Pour les militants, ces six mois de conflit ont constitué un moment fort des trajectoires individuelles, redessinant parfois un profil militant plus éloigné des institutions mais aussi parfois, au moins temporairement, plus engagé dans l'arène électorale. Dans tous les cas, les jeunes étudiants militants et leurs associations ont « fait le politique » pendant quelques mois.

Sur le plan théorique, rendre compte de cette capacité d'un mouvement social de suspendre les règles normales du politique et de le transformer permet d'interroger les conclusions de ceux qui situent l'action collective en-deçà d'autres formes d'action politique, jugées plus efficaces. Cela nous permet aussi d'élargir la discussion à propos des effets des mouvements sociaux. Plutôt que d'évaluer l'action collective protestataire à l'aune des changements dans les politiques publiques, un peu comme on fait de l'évaluation de politiques, il semble plus prometteur d'analyser les changements apportés par l'action dans les dynamiques entre les différents acteurs en présence ou les processus politiques. Certes, il est vrai que la campagne électorale du printemps 2014 a été étonnamment silencieuse sur l'enjeu de l'éducation et que le Parti libéral est revenu avec une confortable majorité au pouvoir. Est-ce que rien n'a changé ? Le mouvement étudiant de 2012 n'aura-t-il été qu'un sursaut contestataire sans 
lendemain? Notre analyse ne permet pas de répondre sans ambages à cette question, mais elle met néanmoins en relief deux éléments qui sont potentiellement porteurs de changement : certains des militants de 2012 ont quitté les bancs de l'école et ont investi d'autres organisations (notamment syndicales et communautaires); ils apportent avec eux une expérience militante hors du commun qui pourrait modifier les règles du jeu à moyen-terme. Par ailleurs, lors du prochain conflit social d'envergure, certaines des réflexes institués en 2012 chez les acteurs collectifs pourraient réapparaître (on peut penser, par exemple, aux recours au système judiciaire par le biais des injonctions ou aux mobilisations citoyennes spontanées). C'est dans ce type de transformation plus difficilement mesurable que l'on pourra à terme prendre la mesure réelle des événements du printemps 2012. 


\section{Références}

Amenta, Edwin, Neal Caren, Elizabeth Chiarello et Yang Su. 2010. « The Political Consequences of Social Movements ». Annual Review of Sociology 36 : 287-307.

Ancelovici, Marcos et Francis Dupuis-Déri, dir. 2014. Un printemps rouge et noir. Regards croisés sur la grève étudiante de 2012. Montréal : Écosociété.

Argument. 2013. « Le peuple selon la CLASSE ». Dossier spécial de la Revue Argument 15 (2). Assemblée Nationale du Québec. 2012. Journal des débats, $39^{e}$ législature, $2^{e}$ session. $<$ http://www.assnat.qc.ca/fr/travaux-parlementaires/journaux-debats.html $>$ (consulté en mai 2013).

Association pour une solidarité syndicale étudiante. 2005. Manifestation de l'ASSÉ pour l'accessibilité, la gratuité, la qualité et le financement de l'éducation. <http://www.assesolidarite.qc.ca/actualite/manifestation-de-lasse-pour-laccessibilite-la-gratuite-la-qualiteet-le-financement-de-leducation/> (consulté en février 2014).

Association pour une solidarité syndicale étudiante. 2014. Présentation. < http://www.assesolidarite.qc.ca/asse/presentation/> (consulté en février 2014).

Baril, Philippe. 2005. La grève de 2005 : Compte-rendu et recommandations. Longueuil : AGECEM.

Bartolini, Stefano. 2005. «La formation des clivages ». Revue internationale de politique comparée 1 (12) : 9-34.

Baumgartner, Frank R. et Christine Mahoney. 2005. « Social Movements, the Rise of New Issues, and the Public Agenda ». Dans Routing the Opposition : Social Movements, Public Policy, and Democracy, dir. David S. Meyer, Valerie Jenness et Helen Ingram. Minneapolis : University of Minnesota Press. 
Bélair-Cirino, Marco. 2012. « Grand tintamarre contre la loi 78-Les casseroles s'en mêlent ». Le Devoir (Montréal), 24 mai, A1.

Bélanger, Éric et Richard Nadeau. 2009. Le comportement électoral des Québécois. Montréal : Presses de l’Université de Montréal.

Bélanger, Pierre. 1984. Le mouvement étudiant québécois : son passé, ses revendications et ses luttes. Montréal : ANEQ.

Brisson, Pierre-Luc. 2012. Après le printemps. Montréal : Poètes de brousse.

Brunelle, Christian, Louis-Philippe Lampron et Myriam Roussel. 2012. « La liberté d'expression en contexte de crise : le cas de la grève étudiante ». Les Cahiers de droit 53 (4) : 831-59.

Burstein, Paul et Sarah Sausner. 2005. « The Incidence and Impact of Policy-Oriented Collective Action : Competing Views ». Sociological Forum 20 (3) : 403-19.

Chabanet, Didier et Marco Giugni. 2010. «Les conséquences des mouvements sociaux ». Dans Penser les mouvements sociaux : Conflits sociaux et contestations dans les sociétés contemporaines, dir. Éric Agrikoliansky, Isabelle Sommier et Olivier Fillieule. Paris : La Découverte, 145-61.

Colas, Valéry. 2008. «L’engagement citoyen des étudiants du Québec ». Dans Les engagements étudiants : des pratiques et des horizons dans un monde globalisé, dir. Françoise Hiraux. Louvain-La-Neuve : Bruylant Academia.

CROP. 2012. La Presse-le conflit étudiant. Montréal : CROP. mai 2012. $<$ http://www.lapresse.ca/html/1230/SONDAGE_CROP_La_Presse.pdf> (consulté en mars 2014). 
Drouilly, Pierre. 2012. « La structure des appuis aux partis politiques québécois, 1998-2008 ». Dans Les partis politiques québécois dans la tourmente, dir. Réjean Pelletier. Québec : Presses de l’Université Laval.

Drouin, Simon. 2012. « Droits de scolarité : Marois prône l'indexation ». Le Soleil (Québec), 3 mai, 9 .

FECQ et FEUQ. 2012. Faire la différence : Plateforme de revendications, élections québécois de 2012. Montréal : FECQ et FEUQ.

Fédération étudiante collégiale du Québec. 2014. Mission. <http://fecq.org/Mission> (consulté en février 2014).

Fédération étudiante universitaire du Québec. 2014. Mission. <http://feuq.qc.ca/a-propos/> (consulté en février 2014).

Fillieule, Olivier et Danielle Tartakowsky, dir. 2008. La manifestation. Paris : Presses de Sciences Po.

Giugni, Marco. 2007. «Useless Protest? A Time-Series Analysis of the Policy Outcomes of Ecology, Antinuclear, and Peace Movements in the United States, 1977-1995 ». Mobilization 12 : 53-77.

Gouvernement du Québec. 2013. « Sommet sur l'enseignement supérieur : des résultats concrets pour s’engager vers une société du savoir ». 26 février. <http://www.mesrst.gouv.qc.ca/ actualites/affichage-des-nouvelles/?tx_ttnews\%5Btt_news\%5D=1229\&cHash= 4c9fdb4d9eabeef556819abbb0877669> (consulté le 26 août 2013).

Grossman, Emiliano et Sabine Saurugger. 2006. Les groupes d'intérêt : Action collective et stratégies de représentation. Paris : Armand Collin. 
Influence Communication. 2013. État de la nouvelle : Bilan 2012. Montréal : Influence Communication.

Isabel, Mariève et Laurence-Aurélie Théroux-Marcotte, dir. 2012. Dictionnaire de la révolte étudiante. Du carré rouge au printemps québécois. Montréal : Tête première.

L’Oreille tendue. 2014. « Des livres sur les grèves étudiantes québécoises de 2012 ». <http://oreilletendue.com/2012/12/03/nouveau-chantier-ecrire-le-printemps-erable/> (consulté le 2 avril 2014).

La Presse canadienne. 2005. « Le ministre Fournier négocierait bientôt avec les étudiants ». Le Soleil (Québec), 12 mars, A8.

La Presse canadienne. 2012a. « Chronologie du conflit étudiant au Québec ». Métro (Montréal), 31 mai. $<$ http://journalmetro.com/dossiers/conflit-etudiant/82780/chronologie-du-conflitetudiant-au-quebec-3/> (consulté le 17 avril 2014).

La Presse canadienne. 2012b. « Marée étudiante dans les rues de Montréal ». Le Devoir (Montréal), 22 mars. $<$ http://www.ledevoir.com/societe/education/345676/journee-degrande-manifestation-etudiante-une-premiere-action-cible-le-port-de-montreal $>$ (consulté le 17 avril 2014).

Lacoursière, Benoît. 2007. Le mouvement étudiant au Québec de 1983 à 2006. Montréal : Sabotard.

Lafrance, Xavier. 2005. « La CASSEE fait le bilan de la grève étudiante—La voie difficile mais nécessaire ». Le Devoir (Montréal), 30 avril, B5.

Lamoureux, Diane. 2013. Le trésor perdu de la politique. Espace public et engagement citoyen. Montréal : Écosociété.

Lavau, Georges. 1981. À quoi sert le parti communiste français ? Paris : Fayard. 
Léger Marketing. 2012a. La grève étudiante. 11 mai, Léger Marketing et Agence QMI. $<$ http://www.leger360.com/admin/upload/publi_pdf/Sondage_JDM_Greve_etudiante_11 _mai_2012.pdf> (consulté le 17 avril 2014).

Léger Marketing. 2012b. La loi qui divise. 21 mai, Léger Marketing et Agence QMI. $<$ http://leger360.com/admin/upload/publi_pdf/Sondage_loi_speciale-21mai.pdf $>$ (consulté le 17 avril 2014).

Léger Marketing. 2013. Intentions de vote provincial au Québec. Montréal : Léger Marketing. Luck, Simon. 2008. « Entre contestation et participation : 1'ambiguïté du rapport au vote des activistes de la gauche libertaire ». Revue française de science politique 58 (2) : 231-56.

Makela, Finn et Sophie Audette-Chapdelaine. 2014. « The Legal Regulation of University Student Associations in Canada ». Education and Law Journal 22 : 267-301.

Makela, Finn. 2014. Recherche sur le droit de grève étudiant au Québec. Montréal : Fédération étudiante universitaire du Québec. <http://feuq.qc.ca/wp-content/uploads/CAI1113_1314_Recherche-Droit-de-grève_VPI_finale_FR.pdf> (consulté le 17 avril 2014). Marcil, Ianik. 2014. «\#biblioencours ». <http://ianikmarcil.com/2012/10/04/biblioencours/> (consulté le 2 avril 2014).

Montigny, Éric et Réjean Pelletier. 2009. «Le pouvoir législatif : le Sénat et la Chambre des communes ». Dans Le parlementarisme canadien : $4^{e}$ édition revue et augmentée, dir. Réjean Pelletier et Manon Tremblay. Québec : Presses de 1’Université Laval.

Nadeau-Dubois, Gabriel. 2013. Tenir tête. Montréal : Lux Éditeur.

Nadeau, Richard et Éric Bélanger. 2013. «Un modèle général d'explication du vote des Québécois ». Dans L'élection québécoise de 2012, dir. Frédérick Bastien, Éric Bélanger et François Gélineau. Montréal : Presses de l’Université de Montréal. 
Parti québécois. 2011. Programme. Montréal : Parti québécois. <http://pq.org/programme> (consulté le 10 mai 2013).

Penafiel, Ricardo. 2012. « Le printemps chilien et la radicalisation de l'action collective contestataire en Amérique latine ». Lien social et politiques 68 : 121-40.

Piven, Frances Fox. 2006. Challenging Authority : How Ordinary People Change America. Lanham, MD : Rowman \& Littlefield.

Poirier St-Pierre, Renaud et Philippe Ethier. 2013. De l'école à la rue : Dans les coulisses de la grève étudiante. Montréal : Écosociété.

Radio-Canada. 2012. « Sondage CROP/Radio-Canada : la loi spéciale n'est pas la solution ». 25 mai. <http:/www.radio-canada.ca/sujet/droits-scolarite/2012/05/25/001-sondage-cropcrise-etudiante.shtml> (consulté le 23 mai 2013).

Riot-Sarcey, Michèle. 2012. « La révolution de 1848 ». Dans Histoire des mouvements sociaux en France de 1814 à nos jours, dir. Michel Pigenet et Danielle Tartakowsky. Paris : La Découverte : 130-52.

Rousseau, Guillaume. 2013. La grève étudiante à la lumière du droit : de l'impasse judiciaire aux solutions politiques. St-Constant : Juripop. <http://www.taceq.org/wpcontent/uploads/2013/02/LA-GRÈVE-ÉTUDIANTE-À-LA-LUMIÈRE-DU-DROIT.pdf> (consulté le 17 avril 2014).

Savard, Alain et Marc-André Cyr. 2014. « La rue contre l'État. Actions et mobilisations étudiantes en 2012 ». Dans Un printemps rouge et noir. Regards croisés sur la grève étudiante de 2012, dir. Marcos Ancelovici et Francis Dupuis-Déri. Montréal : Écosociété, $59-86$. 
Schussman, Alan et Sarah A. Soule. 2005. « Process and Protest : Accounting for Individual Protest Participation ». Social Forces 84 (2) : 1083-1108.

Simard, Marc. 2013. Histoire du mouvement étudiant québécois 1956-2013. Des Trois Braves aux carrés rouges. Sainte-Foy: Presses de l’Université Laval.

Skocpol, Theda. 2003. Diminished Democracy : From Membership to Management in American Civic Life. Norman, OK : University of Oklahoma Press.

Spannring, Rengard. 2012. « Participation and Individualisation : The Emergence of a New (Political) Consciousness ? ». Dans Youth Participation in Europe : Beyond Discourses, Practices and Realities, dir. Patricia Loncle, Morena Cuconato, Virginie Muniglia et Andreas Walther. Bristol : Policy Press, 39-56.

Statistique Canada. 2008. Enquête sociale générale-les réseaux sociaux, cycle 22. Ottawa : Statistique Canada.

Table de concertation étudiante du Québec. 2014. Qu'est-ce que la TaCEQ? $<$ http://www.taceq.org/a-propos-2> (consulté en février 2014).

Theurillat-Cloutier, Arnaud, Alexandre Leduc et Benoît Lacoursière. 2014. « Les racines historiques du Printemps érable ». Dans Un printemps rouge et noir. Regards croisés sur la grève étudiante de 2012, dir. Marcos Ancelovici et Francis Dupuis-Déri. Montréal : Écosociété, 37-58.

Warren, Jean-Philippe. 2008. Une douche anarchie. Les années 68 au Québec. Montréal : Boréal. 


\section{FIGURE 1}

NOMBRE DE MEMBRES EN GRÈVE GÉNÉRALE ILLIMITÉE POUR AU MOINS DEUX SEMAINES PAR AFFILIATION NATIONALE EN 2005

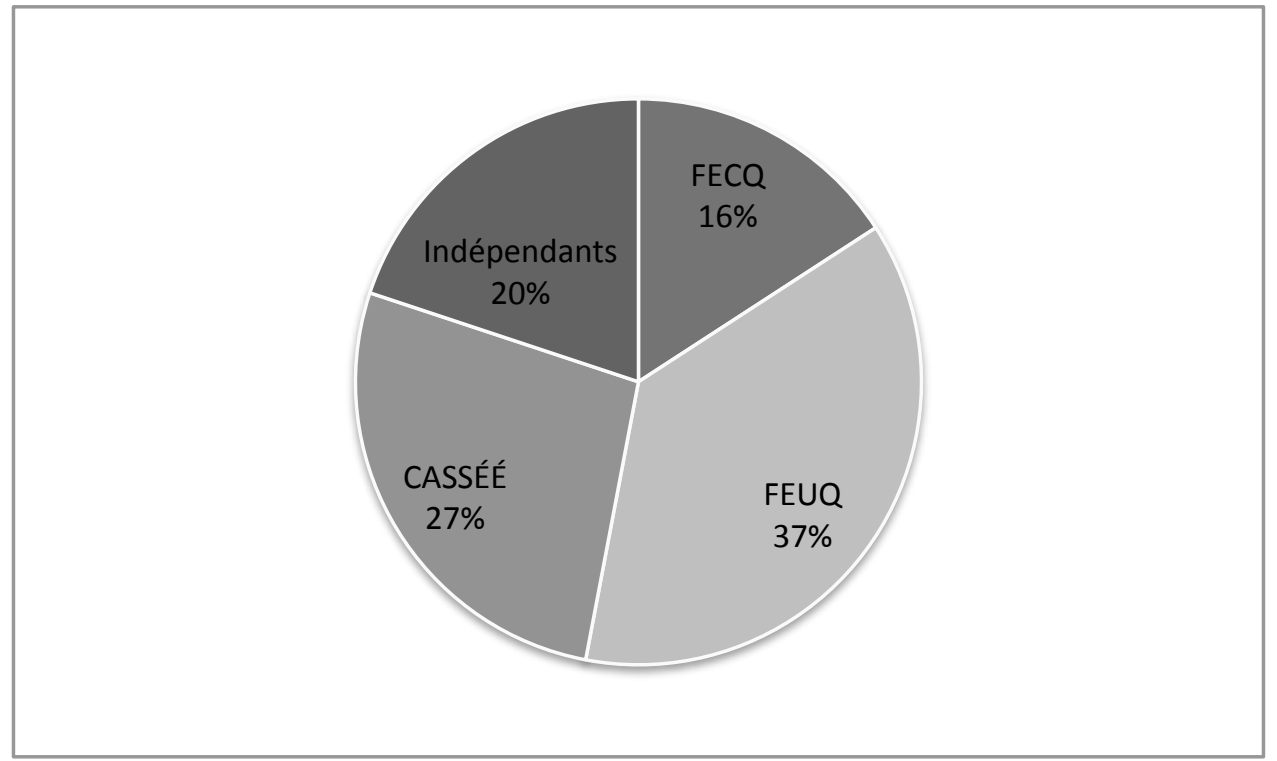

Source : Baril, 2005 et calculs des auteurs. 
FIGURE 2

NOMBRE DE MEMBRES EN GRÈVE GÉNÉRALE ILLIMITÉE POUR AU MOINS DEUX SEMAINES PAR AFFILIATION NATIONALE EN 2012

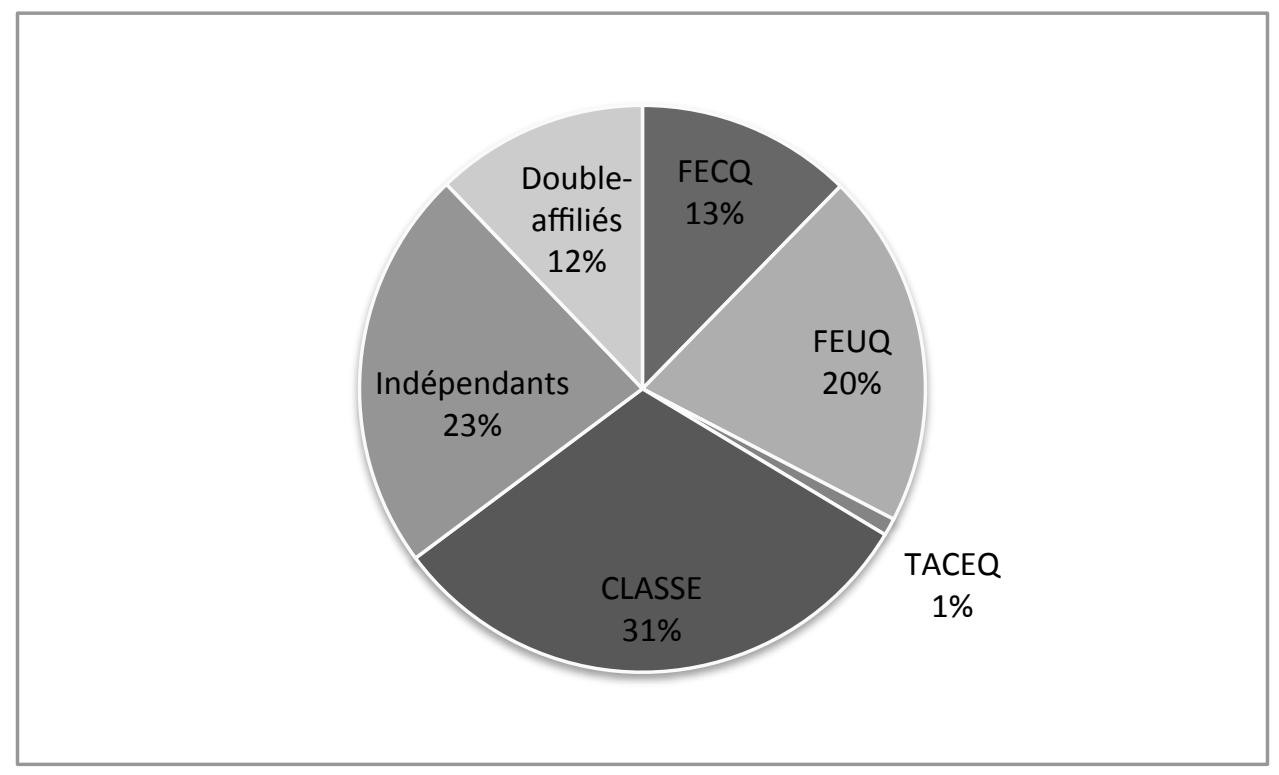

Sources : Données de la FEUQ ; site web www.greve2012.org; site web www.bloquonslahausse.com ; sites web des associations étudiantes ; journaux régionaux ; et calculs des auteurs. 


\section{FIGURE 3}

ÉVOLUTION DE L’APPUI AU GOUVERNEMENT ET AUX ÉTUDIANTS ENTRE LE 23 FÉVRIER ET LE 21 MAI 2012

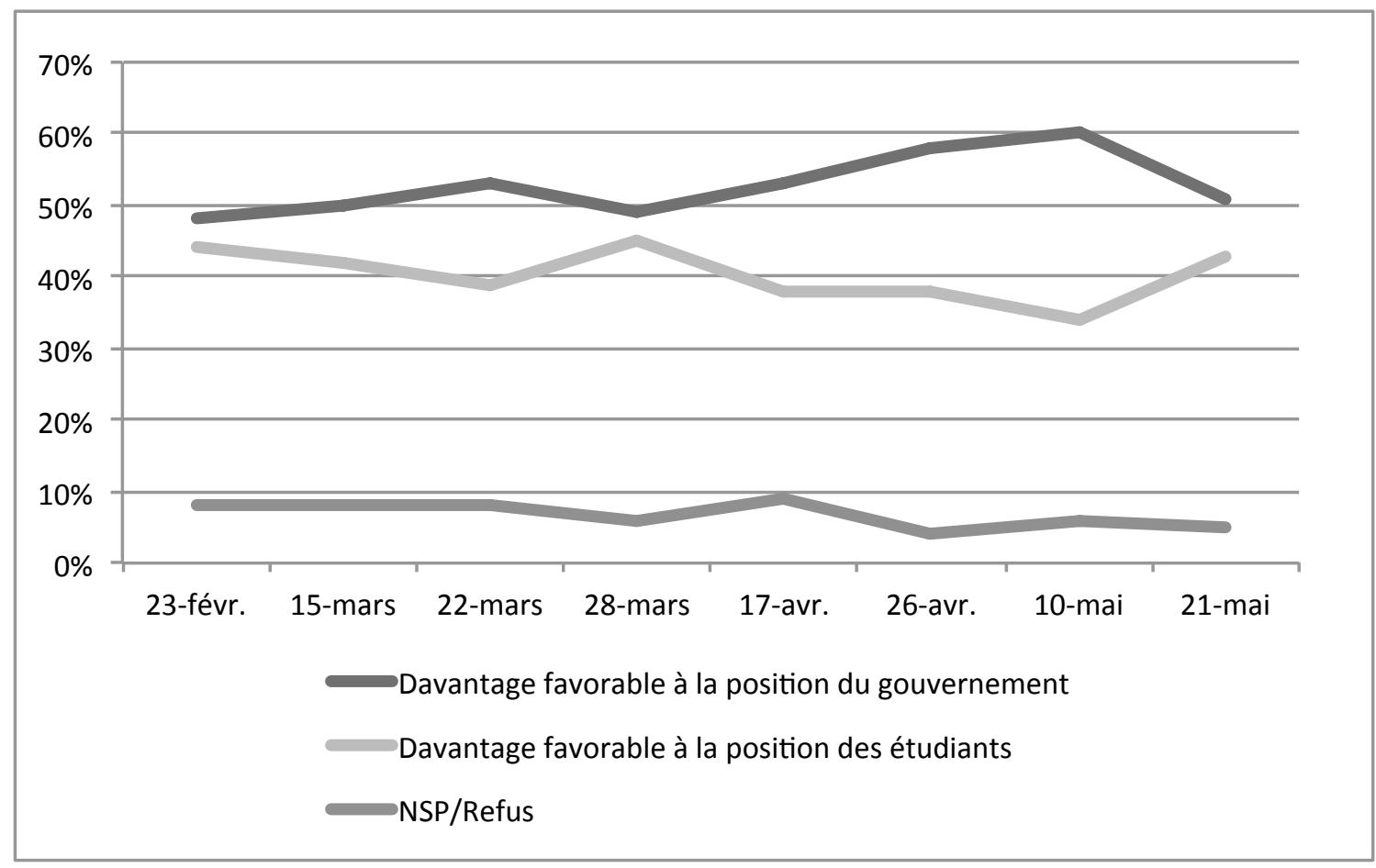

Source : Léger Marketing, 2012a. La question posée est : « Le gouvernement a décidé d'augmenter les frais de scolarité de $325 \$$ par année pendant les cinq prochaines années pour une augmentation totale de 1625 \$. Les étudiants contestent cette décision et demandent le gel des frais de scolarité. Êtes-vous davantage favorable à la position du gouvernement ou davantage favorable à celle des étudiants ? ». 
FIGURE 4

ÉVOLUTION DU TRAVAIL PARLEMENTAIRE DU PQ AU COURS DU CONFLIT ÉTUDIANT

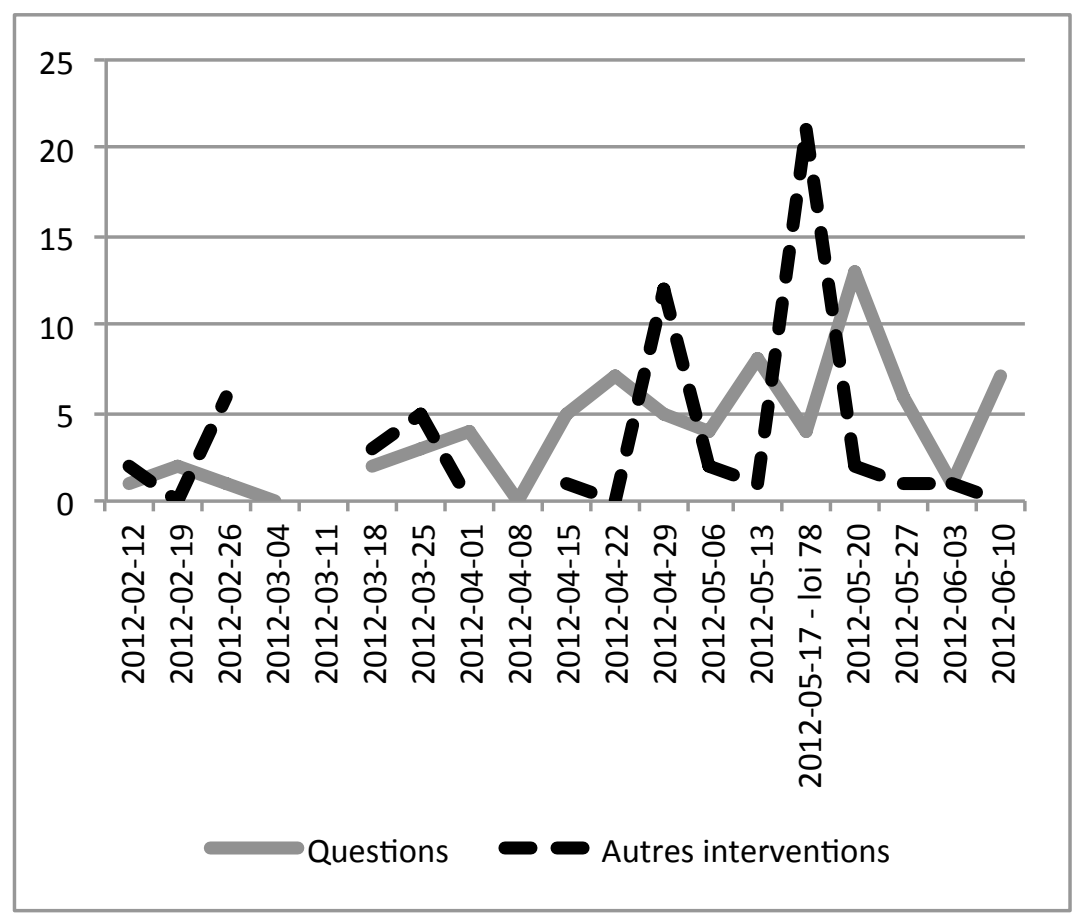

Sources : Assemblée nationale du Québec, 2012. L'Assemblée nationale était en relâche lors des semaines du 4 mars, du 11 mars et du 8 avril. 


\section{Notes}

${ }^{1}$ Pierre Bélanger (1984) trace pour le compte de l'Association nationale des étudiants et étudiantes du Québec (ANEEQ) un portrait du mouvement étudiant de 1960 à 1983. Benoît Lacoursière (2007) s'est penché sur l'histoire du mouvement étudiant de 1983 à 2006 dans une perspective engagée, alors que Valéry Colas (2008) trace un portrait plus historique allant des balbutiements du mouvement étudiant québécois à la grève de 2005. Jean-Philippe Warren (2008) fait quant à lui un bilan des manifestations étudiantes de 1968, et Josianne Millette (2011) s'est penchée sur les associations étudiantes et leurs relations avec les médias durant la grève de 2005. En 2013, Marc Simard a publié une histoire relativement complète du mouvement étudiant de 1956 à 2013.

${ }^{2}$ Les domaines sur lesquels portaient les revendications de la FECQ et de la FEUQ au moment de l'élection provinciale touchaient à l'éducation, les finances publiques, l'économie, l'environnement et l'implication démocratique (FECQ et FEUQ, 2012).

${ }^{3}$ Dans leur chapitre, «La rue contre l'État », Alain Savard et Marc-André Cyr (2014) distinguent également trois phases au conflit qui recoupent largement celles que nous présentons, même si les dénominations sont distinctes. Pour une présentation détaillée des faits, voir leur contribution.

${ }^{4}$ Le caractère spontané de ces manifestations de casseroles, initiées par le biais du réseau social Facebook, rend difficile une compilation précise des manifestations de casseroles. Un groupe de citoyens a toutefois mis en ligne un répertoire des manifestations de casseroles, disponible en ligne au http://on.fb.me/ZbpzRj.

${ }^{5}$ Les autres formations politiques le font, mais de manière nettement plus modeste dû à leur nombre plus restreint de députés, limitant ce faisant le nombre de possibilités d'interventions.

${ }^{6}$ Les questions sont comptées par blocs de questions. Ainsi, est comptabilisé comme un échange une question principale ainsi que toutes les questions complémentaires qui en découlent. On retrouve généralement dans un échange une question principale et deux questions complémentaires ; on ne compte que le nombre de questions principales. 